\title{
Effect of repetitive potassium iodide on elderly rat's thyroid
}

\author{
Dalila Lebsir $^{1,}{ }^{*}$, Elsa Cantabella ${ }^{1}$, Teni Ebrahimian $^{1}$, Dimitri Kereselidze ${ }^{1}$, Stephane \\ Grison $^{1}$, Amandine Sache ${ }^{1}$, Karine Tack ${ }^{1}$, Marc Banderitter ${ }^{1}$, Annick Pech $^{2}$, Philippe \\ Lestaevel $^{1}$, Maâmar Souidi ${ }^{1}$ \\ ${ }^{1}$ Institut de radioprotection et de sureté nucléaire, 92262 Fontenay-aux-Roses, France \\ ${ }^{2}$ Pharmacie centrale des armées, 45000 Orléans, France
}

\section{Abstract}

Background: Nuclear power plant emergencies had often been accompanied by radioactivity release into the environment, thyroid cancer is one of the major health consequences due to the effect of radioactive iodine $\left({ }^{131} \mathrm{I}\right)$ that emits $\Upsilon$ ray and $\beta$ particles resulting in thyroid DNA damage and late onset thyroid cancer. Intake of a single dose of potassium iodide $(\mathrm{KI})$ is recommended to reduce this risk. However in case of prolonged radioiodine release as noticed during Chernobyl and Fukushima accidents, more than one dose of KI may be basic to ensure adequate protection ${ }^{[1]}$. Whereas a single dose of KI is admitted to be safe, knowledge about the effects of repeated KI administration are scarce, few studies demonstrated the potential efficiency of repetitive KI intake in humans ${ }^{[2]}$ and non-human primates ${ }^{[3]}$ without hormonal variations. These studies are relevant in the field of radiation protection and give a base evidence of the possible use of repetitive KI. On the other hand, we have studies on rodents that showed an impact of chronic iodine excess on pituitary thyroid axis function ${ }^{[4]}$. Our previous work on adult male rats demonstrated the safety of repeated administration of KI over 8 days ${ }^{[5]}$. Indeed in the elderly persons KI administration in case of nuclear emergency remains a topic of debate, because of the possible impact in cardiovascular diseases. Thyroid hormones are well-known for their profound effects on cardiovascular function and metabolism; myocardial and vascular endothelial tissues have receptors for thyroid hormones and are sensitive even to subtle changes in the concentrations of circulating pituitary and/or thyroid hormones i.e. subclinical hypothyroidism and hyperthyroidism. It is well established that hyperthyroidism induces a hyper-dynamic cardiovascular state, which is associated with a faster heart rate, enhanced left ventricular systolic and diastolic function whereas hypothyroidism is characterized by the opposite changes. Atrial fibrillation is the most common cardiac arrhythmia in the elderly, the prevalence and incidence increase with advancing age ${ }^{[6]}$. Several interventional trials showed that treatment of subclinical thyroid diseases improves cardiovascular risk factors, which implies potential benefits for reducing cardiovascular events. Objective: The aim of this study is to assess the effects of repeated KI intake on the thyroid function of aged male rats. Methods: A twelve months old male rats were subjected to either KI or saline solution over 8 days. Clinical biochemistry, pituitary and

*Corresponding author: dalila.lebsir@irsn.fr 
thyroid hormones level, and thyroid genes expression were analyzed 30 days after the treatment discontinuation. Findings: urinary assessment shows a subtle increase of some parameters (Creatinin, Uric Acid, Urea, Glucose, Potassium, Sodium and Chlorine), plasma biochemistry reveals a subtle variation of some parameters (an increase of Creatinin, Glucose and phosphorus; and a decrease of Chlorine level). Regarding pituitary-thyroid hormones we get a significant decrease of TSH level without thyroid hormones variation. At the molecular level, we observe a significant increase of TPO $(+100 \%)$, AIT $(+299 \%)$ and $\mathrm{Tg}(+38 \%)$ mRNA expression. On the other hand we get a significant decrease of TSHR (-51\%) mRNA expression. Conclusion and perspectives: Our first results indicate that repeated KI intake affects the clinical biochemistry and the pituitary-thyroid axis function in elderly rats. To go further we are investigating the impact of these variations on the cardiovascular function and its parameters. Cardiac output data, cardiovascular gene expression, oxidative stress and inflammatory analysis are being processed. This study will contribute to the evolution of iodine policy and the harmonization of the current KI guidelines.

\section{Figure}

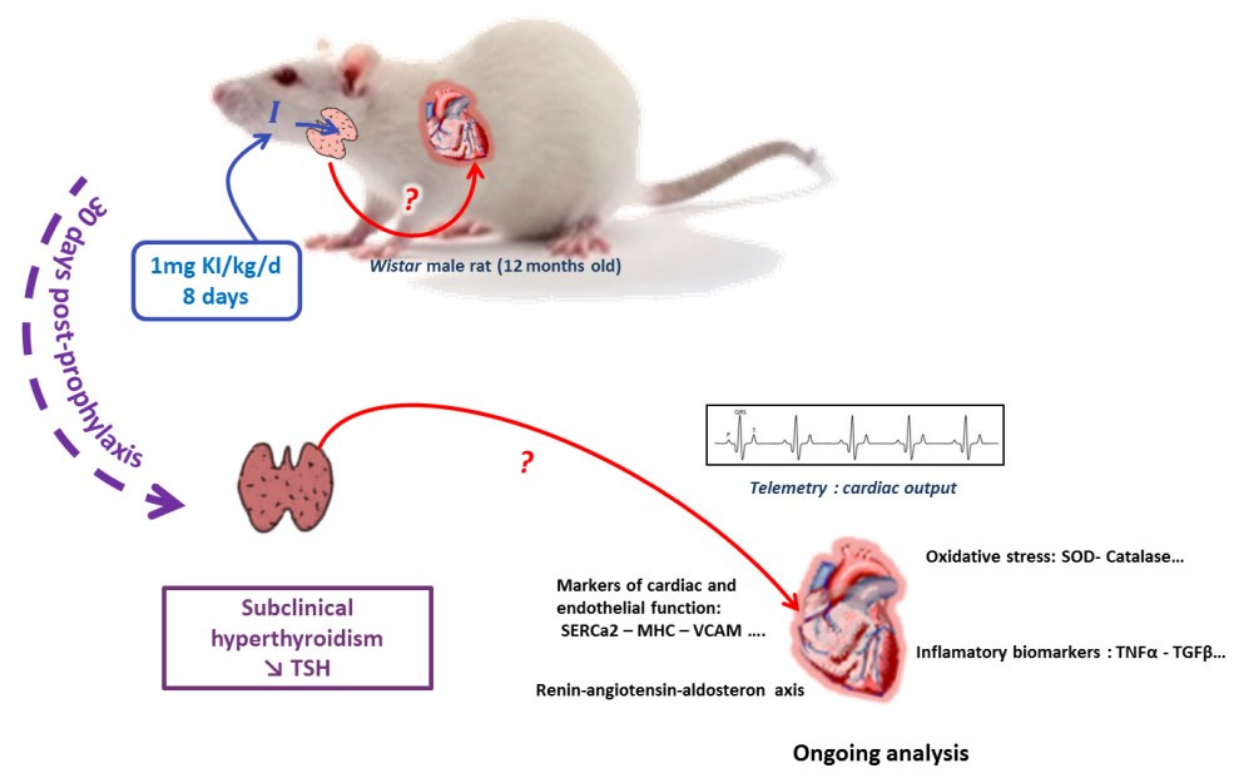

Fig. 1. Impact of repeated KI intake on the thyroid of elderly male rat

\section{References}

1. WHO. ISBN 9789241550185 (2017).

2. Sternthal, E., et al. " N Engl J Med 303(19): 1083-1088. (1980).

3. Noteboom JL., et al. Radiat Res.147(6):698-706. (1997).

4. Calil Silveira J., et al. Thyroid. 26:26. (2016).

5. Lebsir D., et al. Mol Cell Endocrinol.26(18):30077-7. (2018).

6. Delitala AP. Horm Metab Res.49(10):723-31. (2017). 